\title{
A GALAXY AT A REDSHIFT OF 3.215: FURTHER STUDIES OF THE PKS 1614+051 SYSTEM ${ }^{\text {a) }}$
}

\author{
S. DJORGOVSKI ${ }^{\text {b),c) }}$ \\ Harvard-Smithsonian Center for Astrophysics, 60 Garden Street, Cambridge, Massachusetts 02138 \\ Michael A. Strauss \\ Department of Astronomy and Department of Physics, University of California, Berkeley, California 94720 \\ R. A. Perley \\ National Radio Astronomy Observatory, Charlottesville, Virginia 22903 \\ Hyron SPINRAD ${ }^{\text {b) }}$ aNd Patrick MCCARThy \\ Department of Astronomy, University of California, Berkeley, California 94720 \\ Received 9 December 1986; revised 22 January 1987
}

\begin{abstract}
A narrow-emission-line companion of the quasar PKS $1614+051$ was reported earlier as a probable galaxy at a redshift of 3.218 , which would have made it by far the most distant galaxy known at the time. We report here on new radio and optical imaging, and optical and near-IR spectroscopy of the PKS $1614+051$ system. We argue that the data support and reinforce the original interpretation of the companion object as a mildly active galaxy, possibly a marginal Seyfert 2 . The object has a detectable and marginally resolved optical continuum, but was not detected at radio wavelengths. The ionization state is low, and the emission lines are fairly narrow. The improved redshift for the companion, based on the Ly $\alpha$ line alone, is $3.215 \pm 0.002$. New Ly $\alpha$ images show interesting morphology of extended emission-line gas, suggestive of a possible tidal interaction with the neighboring QSO. Two other, fainter objects in the immediate proximity of the QSO may also be associated with the system. If this is the case, we may be seeing a group of galaxies in the early stages of formation.
\end{abstract}

\section{INTRODUCTION}

A galaxy at a redshift greater than three must be an interesting object: it would be very close to the epoch of galaxy formation, and perhaps still forming; it would be among the deepest probes so far of galaxy evolution and cosmology; and its very existence would constrain current scenarios of the origin of large-scale structure and the galaxy formation. The possibility of the detection of one such object was indicated when we applied for the very first time a new technique for finding galaxies at very large redshifts, Lyman $\alpha$ narrowband imaging of distant QSO fields (Djorgovski et al. 1985, hereafter referred to as Paper I). The technique consists of deep CCD imaging of known QSO fields in a narrow band, centered on the redshifted Ly $\alpha$ line, and in a broad band sampling the nearby continuum. Any objects with an "excess" in the Ly $\alpha$ band are then followed up with the slit spectroscopy. The first object found in this way was a narrow-emission-line companion to the quasar PKS $1614+051$, at a redshift of 3.218. It was evident from our spectroscopy and photometry that this object was not a separate QSO itself, nor a particularly bright Seyfert 1 galaxy, nor a gravitationally lensed secondary image of PKS $1614+051$ itself. We proposed in Paper I that it was a mildly active or a starburst galaxy, but with the data at hand at that time we could not dismiss other possibilities, such as a gas cloud ionized by the QSO, or something else.

\footnotetext{
a) Based in part on observations obtained at the Multiple Mirror Telescope Observatory, a joint facility of the Smithsonian Institution and the University of Arizona.

b) Visiting Astronomer, Kitt Peak National Observatory, National Optical Astronomy Observatories, operated by AURA, Inc., under contract with the NSF.

c) Society of Fellows, Harvard University.
}

The technique of Ly $\alpha$ imaging yielded two more similar objects in the field of the gravitational lens MG $2016+112$, at $z=3.27$ (Schneider et al. 1986); it is possible that these are two images of a single, but gravitationally lensed object. Thus the companion of PKS $1614+051$ is not a unique object, nor the only candidate for $a z>3$ (non-QSO) galaxy. Since then, we have searched about fifteen or so additional $z \geqslant 3$ QSO fields for companions with this method, and have found no other objects as luminous in Ly $\alpha$ as the companion of PKS $1614+051$, although it is possible that there are some very faint objects in these fields; a detailed account of that survey will be presented elsewhere. Hu and Cowie (1987) report similar negative results from a search of 11 QSO fields. Apparently, narrow-Ly $\alpha$ objects as luminous as the companion of PKS $1614+051$ are relatively rare, and an upper limit to their frequency is that they may be found in the proximity of one of every 20 or so high-redshift QSO fields. This relative paucity is still consistent with the interpretation of them as luminous galaxies in the upper part of the luminosity function.

The companion of PKS $1614+051$, and the Ly $\alpha$ companion (s) of MG $2016+112$, remain our only solid cases at this time. We need to check whether these objects are indeed galaxies, and learn more about their properties. With this in mind, we undertook a more detailed study of the PKS $1614+051$ system.

\section{OPTICAL IMAGING AND PHOTOMETRY}

Deep CCD images of the PKS $1614+051$ field were obtained with a TI $800 \times 800$ device at the prime focus of the Kitt Peak 4 m Mayall telescope, on the night of 9 April 1986 UT. The seeing had a PSF FWHM of $1.6 \mathrm{arcsec}$, and the sampling was $0.297 \mathrm{arcsec} / \mathrm{pixel}$. Two exposures of $600 \mathrm{~s}$ each were obtained in the $R$ band, and one exposure of $600 \mathrm{~s}$ 
in the $I$ band, in the Kitt Peak Mould-Bessel photometric system. The $I$ exposure was obtained near dawn and through a thin cirrus cover, and will not be used in the present analysis. Two redshifted Ly $\alpha$ images, of 1200 and 1500 s respectively, were obtained with an interference filter (KPNO \#673) whose effective central wavelength at the $4 \mathrm{~m}$ prime focus was $5113 \AA$ and FWHM $\simeq 56 \AA$. The broadband exposures were obtained in the "short-scan" data-taking mode. The telescope was always offset slightly between exposures, in order to improve the flatfielding and to assure the repeatability checks of faint features. The frames were processed and calibrated by the standard techniques. The $R$ band contains the redshifted C IV 1549 emission line, but as we will argue below, the image of the companion is not dominated by it, and represents mainly continuum. Figure 1 shows the portions of the stacked $R$ and Ly $\alpha$ images centered on the QSO. Figure 2 shows the same images processed with a maximum entropy method, in an implementation by M. Irwin (IOA, Cambridge, U.K.), which assumes the correct Poisson noise, and a Gaussian PSF approximation.

Several features can be noted: The companion (designated here as " $\mathrm{A}$ ") is marginally resolved in the $R$ band, and its
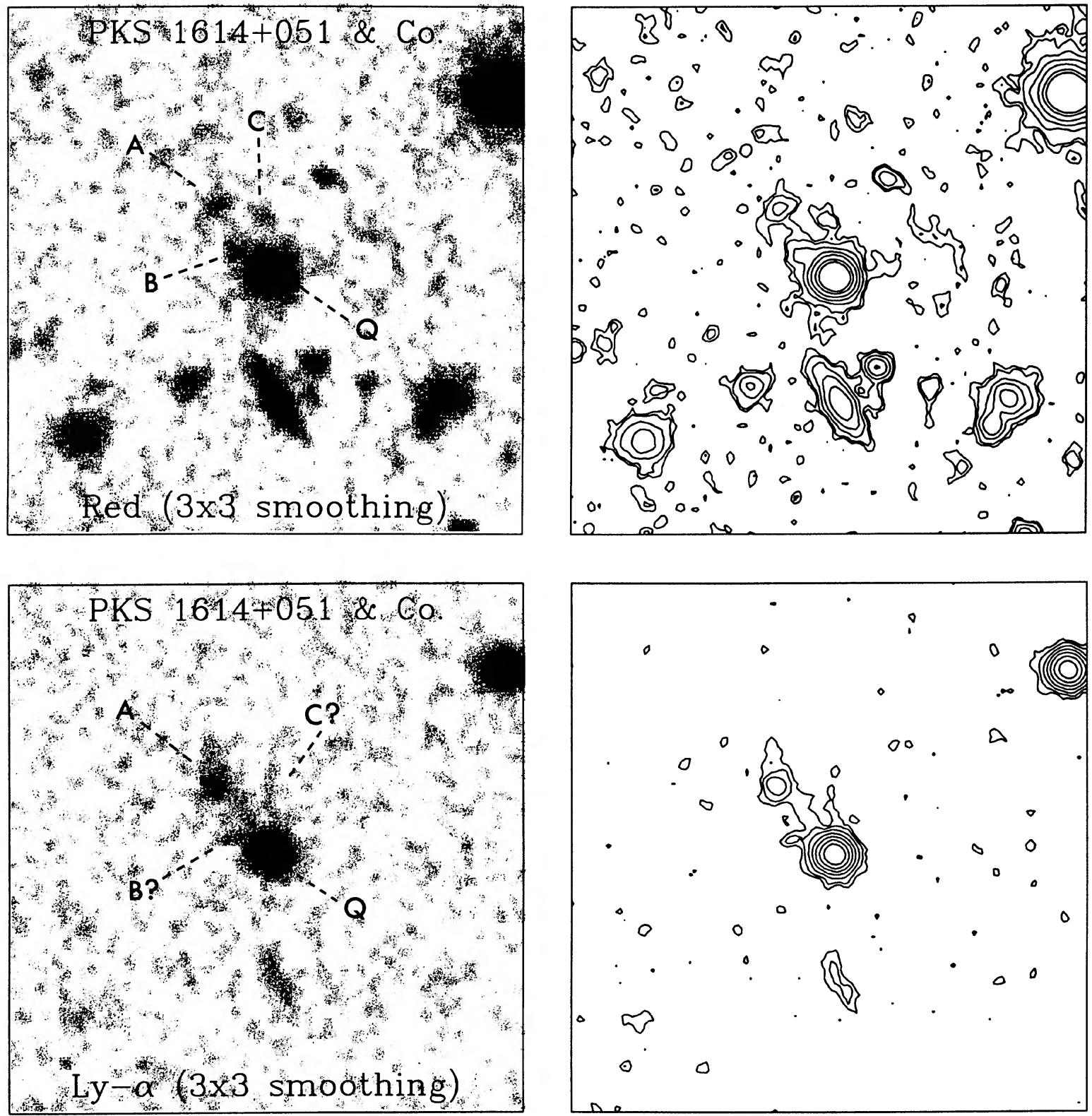

FIG. 1. Direct images of the PKS $1614+051$ field, in the $R$ (top) and Ly $\alpha$ (bottom) bands. The fields shown here are 38 arcsec square, with north at the top and east to the left. The images were smoothed with a $3 \times 3$ pixel box filter in order to suppress the noise. The QSO ("Q") and its three faint companions ("A", "B", and "C") are labeled in the gray-scale panels. The contours have roughly logarithmic spacing. Note that the foreground galaxies (possibly a foreground cluster) to the south of the QSO effectively disappear in the Ly $\alpha$ image. 

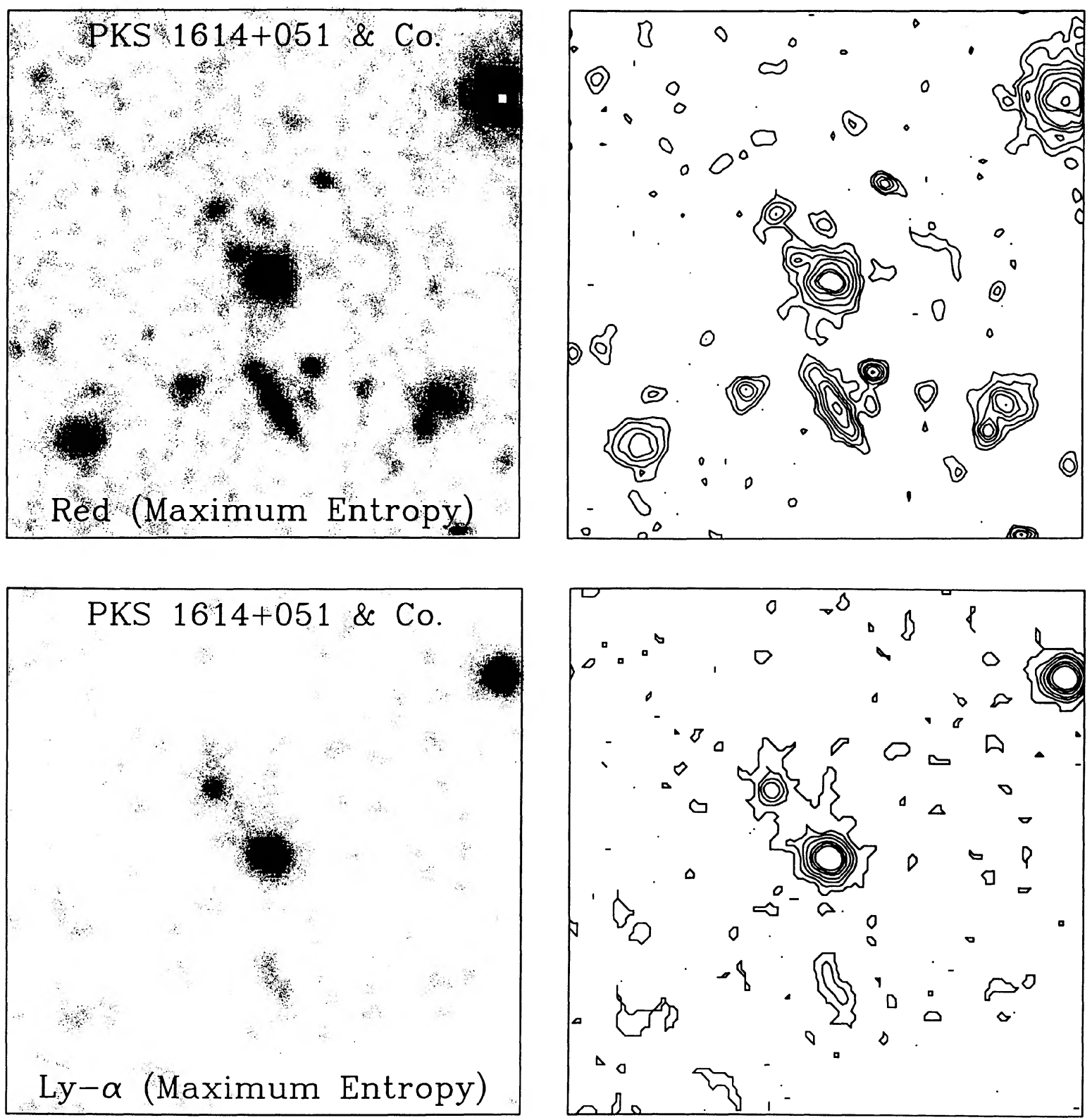

FIG. 2. Same as Fig. 1, but after a maximum entropy processing, which suppresses the noise and increases the contrast. The features indicated in the "raw" data images shown in Fig. 1 are now clearly seen. The faint objects A, B, and C, and the extended Ly $\alpha$ features, are repeatable (but, of course, noisier) in the separate exposures from which these stacks were produced.

core appears stellar in the Ly $\alpha$ band. However, there is clearly an extended Ly $\alpha$ bridge between it and the QSO, and a further extension of the line emission beyond $\mathrm{A}$ to the north. There is also a marginally detected tail of Ly $\alpha$ emission going north from the QSO. On the continuum images, there are two fainter objects, denoted "B" and "C," which may possibly be associated with some of the Ly $\alpha$ emission near the QSO. However, their "Ly $\alpha$ excess" is nowhere near that of the originally discovered companion, A. All these features are repeatable from frame to frame.

Photometry was done using the NGC 2419 and NGC 4147 standard fields (Christian et al: 1985). The red magni- tudes and relative positions of the objects are summarized in Table I. The improved coordinates (Epoch 1950.0) for the QSO are: $\alpha=16^{\mathrm{h}} 14^{\mathrm{m}} 09$ s.08, $\delta=+05^{\circ} 06^{\prime} 54^{\prime \prime} .92$, with errors of 0.1 arcsec in each coordinate (Morabito 1986, private communication). In order to interpret the angular scale of these data in physical units, we note that at the redshift of the PKS $1614+051$ system, in a $\Lambda_{0}=0$ Friedman cosmology, 1 arcsec corresponds to $6.86 h^{-1} \mathrm{kpc}$ if $\Omega=0$, or to 3.54 $h^{-1} \mathrm{kpc}$ if $\Omega=1$. Thus, the projected separations of these objects are of the order of a few tens of kpc, not unlike those in compact galaxy groups, or between our galaxy and the Magellanic Clouds. 


\section{RADIO IMAGING}

Radio observations of the field were made with the VLA on 28 March 1986, when the array was in its maximum resolution A configuration. About $86 \mathrm{~min}$ of data were taken at both 6 and $20 \mathrm{~cm}$ wavelengths. Both available IF pairs were utilized, resulting in an effective bandwidth of $100 \mathrm{MHz}$ at each band. The flux-density scale was established by short observations of 3C 286. Because the quasar PKS $1614+051$ is itself a strong, nearly unresolved radio source, no independent phase calibration was required, and subsequent processing used the quasar as a phase and amplitude reference. This processing was done in two steps. First, a standard antenna-based self-calibration was done, using the current image of PKS $1614+051$. Two passes of self-calibration were made, the first correcting the phases only, and the second correcting both the amplitudes and the phases. These steps produced images with dynamic ranges of 3000-5000, at about the expected level for the quantity of data taken. Studies have shown that further improvement in dynamic range (and hence in detectability of weak sources in fields dominated by strong emission) requires correction for the socalled closure errors-that is, baseline-based errors which cannot be corrected by antenna-based calibration techniques. Since PKS $1641+051$ is a strong, isolated source with a simple structure, it can be used to estimate these errors. The source removal and the estimation of these errors constituted the second step of our processing. The resulting images had noise levels at or close to the levels expected from a random Gaussian process. More complete descriptions of these procedures are given by Cornwell (1986) and Perley (1986).

Figure 3 shows the resulting 6 and $20 \mathrm{~cm}$ maps of the field. No emission is seen in the vicinity of the companion in either band. The residual $1 \sigma$ noise levels in these images are 33 and $63 \mu \mathrm{Jy}$ per beam, respectively (the higher noise level in the $20 \mathrm{~cm}$ image is believed to be primarily due to the effects of distant sources that were not properly modeled in the selfcalibration procedure). The radio structure of the quasar is shown to be double, with a strong, unresolved core with an inverted spectrum $(\alpha=0.8)$, identified with the optical QSO, and a one-sided weak secondary (a jet?), with a steep spectrum ( $\alpha=-1$ ), located 0.8 arcsec away from the core, at P.A. $=-30^{\circ}$. This structure is entirely normal for radioloud quasars, being detected in at least $50 \%$ of all such objects (Perley 1982a,b).

The absence of detected radio emission from companion A enhances the difference in physical nature between it and the QSO, but it does not argue against a low-luminosity active nucleus: many Seyfert galaxies are "radio quiet." Our detection limits correspond to a rest-frame power of the order of $10^{24} \mathrm{~W} \mathrm{~Hz}^{-1}$, assuming an intermediate Friedmann cosmology with $H_{0}=75$ and $q_{0}=0.2$, and a radio powerlaw index $\alpha=-0.7$. Most low-redshift Seyferts are fainter in the radio than that (Ulvestad and Wilson 1984). The direction of the secondary radio component (tentative jet) is almost perpendicular to the direction towards companion $\mathrm{A}$, and is also far from the directions of the two other faint objects discussed above, B and C. The companions are clearly not high-redshift cases of jet-galaxy interactions ("Minkowski Objects"; cf. van Breugel et al. 1985, or Brodie et al. 1985).

\section{OPTICAL SPECTROSCOPY}

Our earlier low-resolution CCD spectra (Paper I) indicated that the emission lines in the companion $\mathrm{A}$ are re- solved, with intrinsic widths of some hundreds of $\mathrm{km} \mathrm{s}^{-1}$, but with the error bars comparable to the measured width. Interpretation of the nature of the companion A depends strongly on the exact value of this line broadening. In order to measure the intrinsic widths, spectra with better sampling are needed, and we specifically observed companion A and the QSO with the MMT'Intensified Reticon Spectrograph for that purpose, on the nights of 4 and 5 May 1986 UT. We used the 300 lines/mm grism, with a sampling of about 2 $\AA$ /channel, that is, 2-3 times the sampling of our low-resolution CCD spectra. The apertures used were $2 \times 3$ arcsec slitlets, and the seeing was typically $1.5 \operatorname{arcsec}$ (FWHM). The total integration was $90 \mathrm{~min}$. The Ly $\alpha$ line was the only one detected in the companion A with a sufficient $\mathrm{S} / \mathrm{N}$ for this analysis. A Gaussian fit to the line core gives a $\sigma$ of $6.1 \pm 0.2$ $\AA$ for companion A. In order to measure the instrumental broadening, several bright, uncontaminated lines were measured from the $\mathrm{Hg}-\mathrm{He}-\mathrm{Ne}-\mathrm{Ar}$ comparison spectra, and yielded a mean and a median $\sigma$ of $5.3 \AA$, with a scatter of 0.5 $\AA$. This corresponds to an intrinsic broadening of 3.0 $(+1.1,-1.9) \AA$, or $180(+60,-110) \mathrm{km} \mathrm{s}^{-1}$. In other words, the core of the Ly $\alpha$ line in companion A is almost unresolved, and certainly has a small intrinsic velocity width. This is illustrated in Fig. 4 . We note that it is possible that the Ly $\alpha$ line has broad wings, but the low S/N and the possible presence of contamination by scattered light from the nearby QSO make it impossible to be sure. It is also our impression that the carbon lines may be slightly broader than $\operatorname{Ly} \alpha$, but our data are still too noisy for a good measurement. The improved redshift for the companion $\mathrm{A}$, based on the Ly $\alpha$ line only, is $z=3.215 \pm 0.002$.

Relatively narrow Ly $\alpha$ emission argues against a dominant active nucleus, but the second important test is to estimate the degree of ionization in companion A from the emission-line ratios. We obtained additional CCD spectra of both the QSO and the companion A with the Cryocam low-resolution spectrograph on the KPNO $4 \mathrm{~m}$ telescope. We obtained one $4000 \mathrm{~s}$ exposure on the night of 10 May 1986 UT in the visual-red spectral region, calibrated with the spectrum of the standard star BD $+33^{\circ} 2642$ (Stone 1977), and one $3600 \mathrm{~s}$ exposure on the night of 11 May 1986 UT in the near-IR region, calibrated with the spectra of the standard stars Feige 34 and BD $+25^{\circ} 3941$. Both exposures had the spectrograph slit oriented at P.A. $=40^{\circ}$, in order to include both the QSO and companion A. The seeing was relatively poor in the 10 May (visual) data, and the results did not supersede our previous spectroscopy from Lick Observatory (Paper I). However, the data from 11 May were good, and we did measure the (C IV 1549)/(C III] 1909) line ratios of $1.0 \pm 0.1$ for companion $\mathrm{A}$, and 2.55 for the QSO. We recall from Paper I that the (C IV 1549)/(Ly $\alpha$ 1216) line ratios are 0.28 and 0.51 , respectively. Thus, companion $\mathrm{A}$ has a considerably lower ionization state than the QSO.

Finally, we detected a definite continuum between the emission lines in companion $\mathrm{A}$, as illustrated qualitatively in Fig. 5. We show here the relative intensity cuts along the slit, produced by adding a number of CCD rows in each of three bands: two narrow ones containing the carbon emission lines, and a wide band spanning the continuum between them. The cuts are shown both for the PKS $1614+051$ system ( $\mathrm{Q}$ and $\mathrm{A}$ ) and a comparison star whose exposure was obtained several minutes after the PKS $1614+051$ frame and was processed in an identical way. Note that in the companion $\mathrm{A}$ the continuum appears to be more resolved than the line emission, in agreement with our direct exposures, 

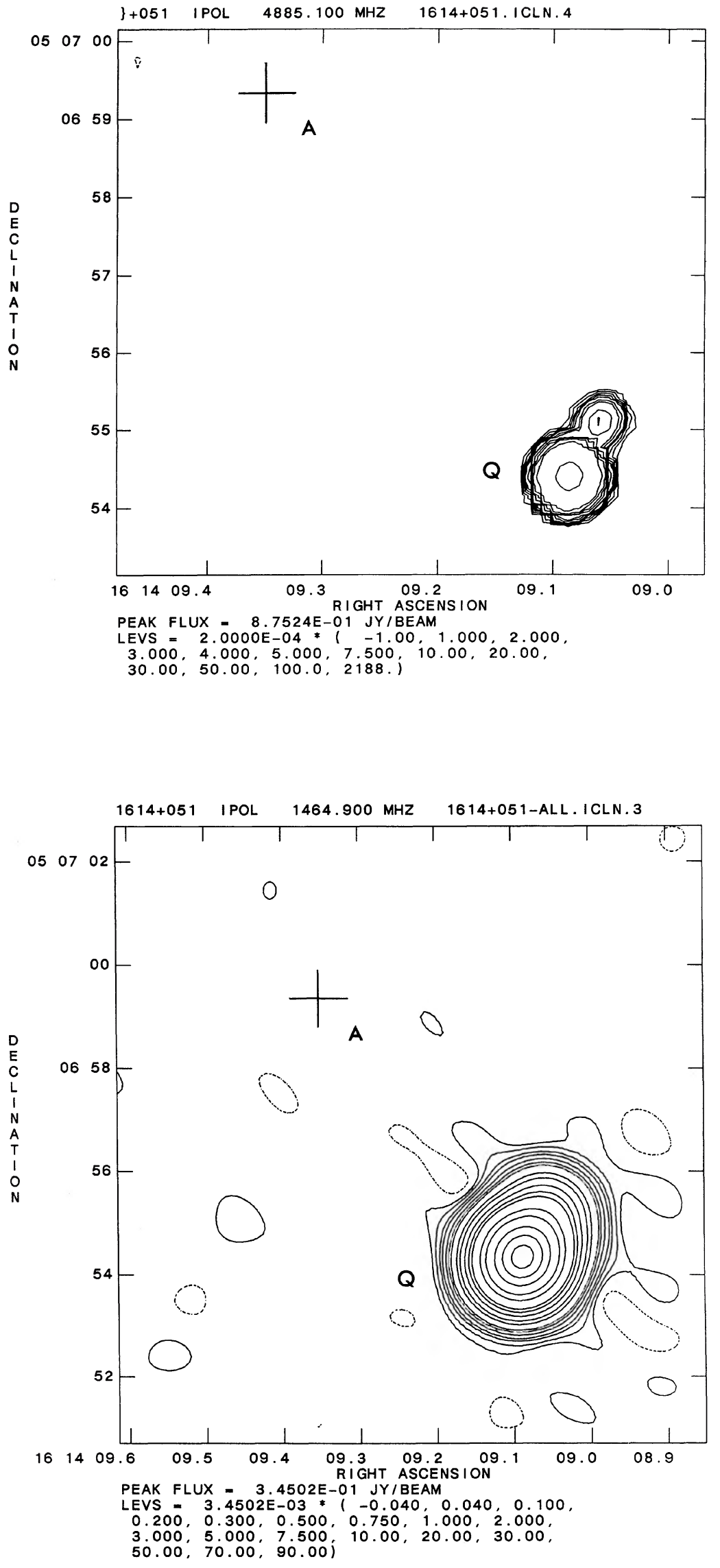

FIG. 3. VLA maps of the PKS $1614+051$ system, at 6 (top) and $20 \mathrm{~cm}$ (bottom). The position of companion $\mathbf{A}$ is labeled with a cross $(+)$. The QSO with the tentative jet is the source at the lower right. 


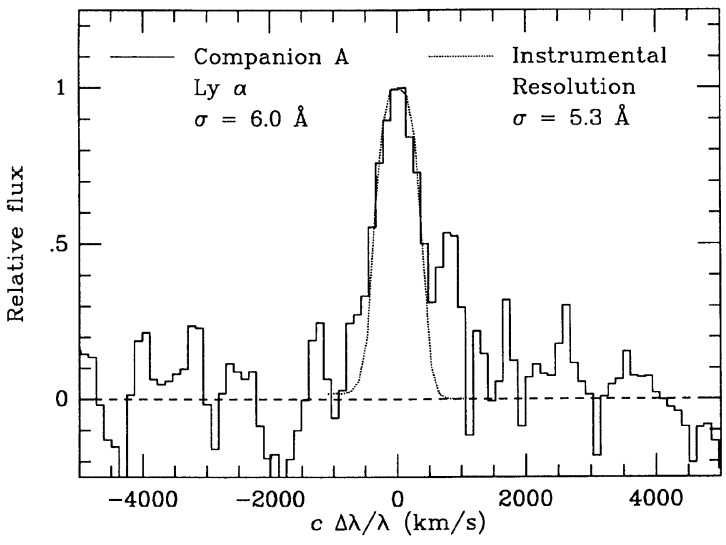

FIG. 4. Zoom-in on the Ly $\alpha$ line in the MMT spectrum of companion $\mathrm{A}$. The dotted line indicates the instrumental resolution. The line core is only marginally broader than the comparison line, but there may be extended wings, possibly due to scattered light from the QSO. shown in Figs. 1 and 2; corresponding features are absent in the cuts from the comparison-star frame. The data are well in the linear regime of the CCD, and there is no danger of an artifact caused by poor charge transfer in the CCD, saturation, or any other known instrumental effect.

The morphology of companion A in these cuts again suggests that the line emission has mostly or entirely a nuclear origin, whereas the resolved continuum may be starlight. The spectroscopic extraction of the continuum is very difficult in the presence of a much brighter QSO nearby, and we cannot measure the equivalent widths of the emission lines with any certainty. We can, however, estimate a rough spectroscopic magnitude in the region of interest, by summing up the counts in the regions that are free from the emission lines: we obtain spectroscopic $R_{\text {cont }} \simeq 24 \pm 0.5$, in excellent agreement with our direct photometry. Thus, we estimate that the C IV 1549 line probably does not contribute more than 0.5 mag to the $R$ band magnitude (Table $I$ ). All this is also in good agreement with our earlier data obtained at Lick (Paper I). The very existence of the optical continuum (which may well be stellar in origin) is an important fact, as it argues
PKS 1614+051

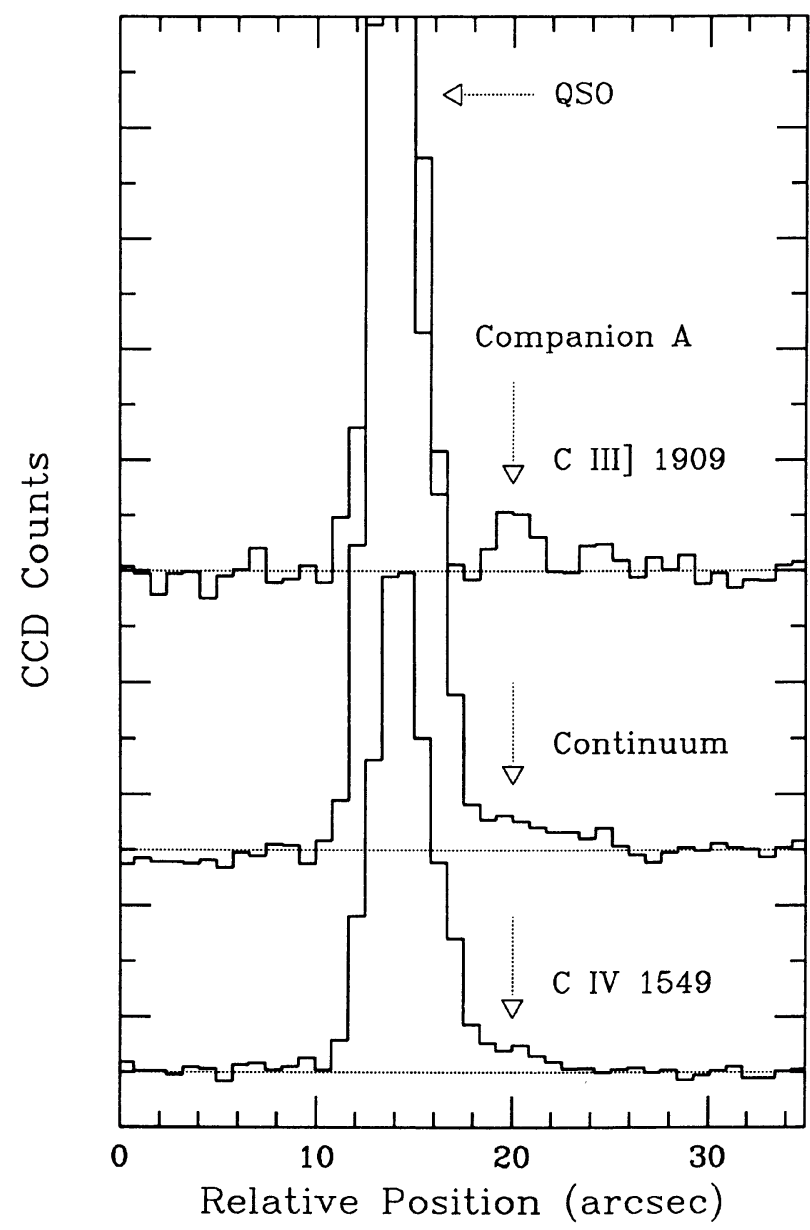

Comparison

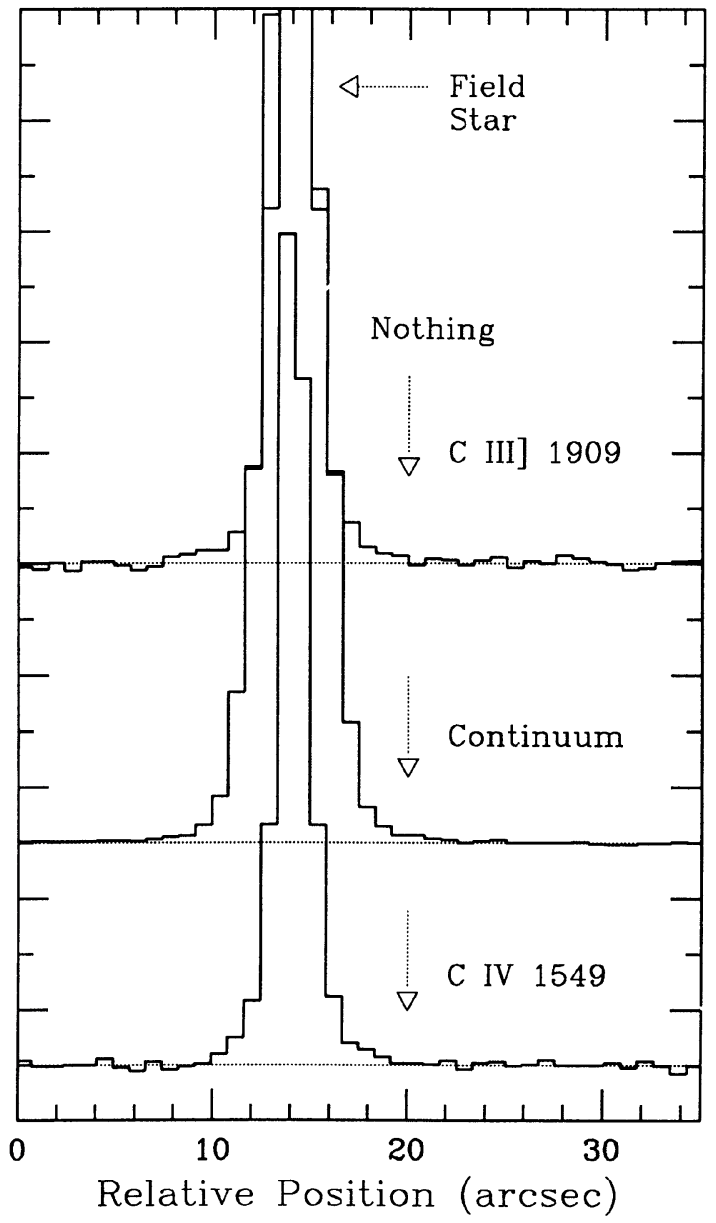

Fig. 5. Spatial intensity profiles, obtained from flattened, sky-subtracted spectroscopic CCD frames, obtained at Kitt Peak on 11 May 1986 UT. These intensity cuts each represent a number of coadded CCD rows, and have arbitrary vertical scalings. The left-hand panel shows the data on the PKS 1614 + 051 system, and the right-hand panel shows equivalent cuts from a frame of a comparison star, processed in the same way; in each of the three bands, the cuts for the QSO and the comparison star are scaled as to have approximately the same areas. The dotted lines indicate the zero level for each cut. The $\mathrm{C}$ IV line in companion A may be slightly defocused, but the continuum is mostly from a well-focused part of the chip, and the extended morphology of companion $\mathrm{A}$ is real. 
TABLE I. Photometry and offsets from QSO.

\begin{tabular}{llcc}
\hline \hline Object & $R$ & $\Delta \alpha_{\mathrm{OBJ}-\mathrm{QSO}}$ & $\Delta \delta_{\mathrm{OBJ}-\mathrm{QSO}}$ \\
\hline QSO & 18.98 & 0 & 0 \\
Comp. A & 23.6 & +4.05 & +4.85 \\
Comp. B & 24.2 & +2.6 & +1.6 \\
Comp. C & 24.6 & +0.5 & +4.1 \\
\hline \hline
\end{tabular}

Notes to TABLE I

The photometric error is $\leqslant 0.1 \mathrm{mag}$ for the QSO, $\sim 0.5 \mathrm{mag}$ for the fainter objects A, B, and C.

The positional offsets are given in arcsec, and are uncertain by $\sim 0.1 \mathrm{arcsec}$. Objects A, B, and C are all to the north and east of the QSO.

against the possibility that companion $\mathrm{A}$ is simply a cloud of ionized gas.

\section{CONCLUSIONS}

We believe that the new data confirm and reinforce our original interpretation of companion $\mathrm{A}$ as a mildly active galaxy. It does have a faint continuum, detected in both direct imaging and slit spectroscopy, which seems to be distributed spatially differently from the emission-line gas. In the absence of any detectable radio emission, this continuum probably represents starlight. The spectroscopic signature (narrow linewidths, low ionization) is that of a marginal Seyfert, although it is possible that some of the line emission (Ly $\alpha$ in particular, possibly some C III]) is excited by the radiation field of the QSO (Filippenko 1985), or the UV continua of young stars if the star formation is still very active in the object. The $\mathrm{C}$ IV line would be difficult to ionize by a dilute radiation field, it appears to be broader than $\mathrm{Ly} \alpha$, and it almost certainly originates in an active nucleus. All this argues against the interpretation of companion $\mathrm{A}$ as a luminous gas cloud ionized by the QSO, similar to the lowredshift systems detected by Stockton and MacKenty (1983). A possible low-redshift analog of this system may be the QSO PKS 2135 - 147 and its emission-line companion (Stockton 1982).

$\mathrm{Hu}$ and Cowie (1987) present independent imaging of this system, obtained with the CFH telescope at Mauna Kea. Companion $\mathrm{A}$ is barely detected in their stack of several $R$ band exposures (which seems to cause a heavy readout-noise penalty), which has a total integration of $8 \mathrm{~min}$, but they did detect the Ly $\alpha$ "bridge" between the companion and the QSO, as well as some of the fainter objects. They conclude from their marginal detection of the continuum in object $A$ (in our notation) that it is probably a gas cloud, ionized by the QSO, a conclusion diametrically opposite to ours. Their interpretation is heavily based on the apparent lack of continuum suggested by their red imaging data. Unfortunately, they used different filters than we did, and expressed their results in $A B_{v}$ magnitudes, computed in 2.4 arcsec square apertures, all of which makes direct comparisons somewhat difficult. However, a simple visual comparison of their $R$ band contour map with the one shown in our Fig. 1 suggests that our images have a considerably higher S/N. A part of the discrepancy may be hidden in the optimistic photometric error bars that they quote for the fainter objects $(0.2 \mathrm{mag})$ and which should be compared with our estimates $(0.5 \mathrm{mag}$, from apparently better-quality data, and obtained by a careful PSF fitting and subtraction). Thus, modulo the differences in the photometric systems and methods, we believe that our higher $\mathrm{S} / \mathrm{N}$ images and the long-slit spectroscopy provide more persuasive evidence for a continuum in companion A.

The spectrum of companion $A$ is not unlike those of the high-redshift 3CR radio galaxies (Spinrad et al. 1985; Spinrad and Djorgovski 1984), and it is argued elsewhere that most of the light from those galaxies comes from rapidly evolving stellar populations at large redshifts (Djorgovski $e t$ al. 1987). Some luminosity evolution is also implied here: the distance modulus to the system, in a $H_{0}=75, q_{0}=0.2$, $\Lambda_{0}=0$ Friedman cosmology, is $(m-M)=46.84$, and even at 24 mag in the rest-frame UV, companion A must be a fairly luminous galaxy. In other words, the optical and spectroscopic properties of companion $\mathbf{A}$ are similar to those of the known, evolving $3 \mathrm{CR}$ galaxies at $z \sim 1-2$, even though it is radio quiet.

The morphology of the system as shown in Ly $\alpha$ emission is suggestive of a tidal interaction, regardless of how the gas is ionized. Evidence for tidal interactions is often indicated in the low-redshift QSO-galaxy associations, and is even proposed as a possible trigger of the QSO activity (cf. the review by Hutchings 1983, and references therein). Possible low-redshift examples of such phenomena are discussed by Hutchings et al. (1986) and Shara et al. (1985), the latter (quasar 4C 18.68) involving a "tidal tail" of extended emission-line gas, reminiscent of the features found in our Ly $\alpha$ images. Another interesting system is the QSO 3C 275.1, which is embedded in a cloud of ionized gas and is a brightest cluster member (Hintzen and Romanishin 1986). The two cases discussed by Stockton and MacKenty (1983), 3C 249.1 and Ton 202, were interpreted by these authors as being results of tidal interactions, which possibly triggered or refueled the QSO activity. It should be pointed out that the emission-line gas clouds detected by Stockton and MacKenty are not obviously galaxies themselves, but may be residuals of a swept material ionized by the collisions and/or the radiation field of the QSO. In the PKS $1614+051$ system, we may be seeing such a fateful interaction occurring at the epoch when quasars first appear in large numbers. In any case, the projected separation and the velocity difference between the QSO and companion $\mathrm{A}$ are consistent with a tidal encounter.

A tentative association of faint objects $B$ and $C$ with the system at $z=3.21$ is an intriguing possibility. One possible check of this hypothesis, which could be done with currently existing technology, is deep, good-seeing imaging in several narrow bands covering Ly $\alpha$ and the continuum near it-in a sense, a crude imaging spectroscopy. Interpretation of the PKS $1614+051$ system as a young and possibly still forming group is also discussed by $\mathrm{Hu}$ and Cowie (1987). Tyson (1986) claims an excess of faint galaxies in the fields of $z \sim 1-$ 1.5 QSOs, and suggests that this may be evidence for both clustering and luminosity evolution at these redshifts. We may be seeing a similar effect here, but at $z>3$.

A galaxy at $z>3$ is quite young, independent of the formation redshift $z_{\mathrm{gf}}$; for example, in a $H_{0}=75, q_{0}=0.2$, $\Lambda_{0}=0$ Friedman cosmology, for $z_{\mathrm{gf}}=5$, the age of the PKS $1614+051$ system is only $0.57 \mathrm{Gyr}$, or $6.2 \%$ of the present galaxian age, or, if $z_{\mathrm{gf}}=10$, the age is $1.08 \mathrm{Gyr}$, or $11.1 \%$ of the present age. Increasing the sample of such extremely distant galaxies is well worth the effort, as they are clearly just past the formation stage and may even be classifiable as primeval galaxies. On the other hand, the presence of wellformed galaxies (not only quasars, which may be dismissed as "peculiar"), and possibly even galaxy groups or clusters, 
at $z>3$ is an important constraint for the theories of largescale structure and galaxy formation. Both hot and cold dark matter pictures, at least in their simplest forms, would prefer that the bulk of the galaxy formation occur at lower redshifts, $z_{\mathrm{gf}} \sim 1-2$, and this may be a difficulty. It is possible, however, that some galaxy formation does extend to red-

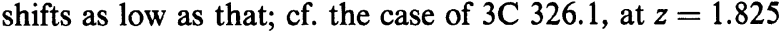
(McCarthy et al. 1986; and in preparation).

It is thus now possible to study galaxies at $z>3$ from the ground in some detail. In the future, high-resolution imaging with the Hubble Space Telescope will give us valuable morphological information and further hints about the nature of these objects, and a better understanding of the whole PKS $1614+051$ system. Continuum spectroscopy of these objects, and the companions of MG $2016+112$, with the $10 \mathrm{~m}$ class telescopes of the next generation should provide us with unique insights into the stellar populations and the physical state of very young galaxies and clusters at large redshifts, and the processes that caused the appearance of quasars at those early epochs.

We are very thankful to the staffs of the Kitt Peak, VLA, and MMT Observatories for their help in obtaining the data used in this work. We also thank Susan Tokarz for her help in reducing the MMT spectra. The radio data were obtained with the Very Large Array, NRAO, which is operated by the Associated Universities, Inc., under a contract with the NSF. We thank L. Cowie and E. Hu for stimulating conversations and communications of their results before publication. The paper benefited from a constructive refereeing by A. Stockton. S. D. acknowledges partial support from Harvard University. H. S. was supported in part by NSF grant AST85-13416. M. A. S. acknowledges the support of an NSF Graduate Fellowship.

\section{REFERENCES}

Brodie, J., Bowyer, S., and McCarthy, P. (1985). Astrophys. J. Lett. 293, L59.

Christian, C., Adams, M., Barnes, J., Butcher, H., Hayes, D., Mould, J., and Siegel, M. (1985). Publ. Astron. Soc. Pac. 97, 363.

Cornwell, T. J. (1986). In Synthesis Imaging, NRAO Workshop No. 13, edited by R. Perley, F. Schwab, and A. Bridle (NRAO, Green Bank, WV).

Djorgovski, S., Spinrad, H., and Dickinson, M. (1987). Astrophys. J. (submitted).

Djorgovski, S., Spinrad, H., McCarthy, P., and Strauss, M. (1985). Astrophys. J. Lett. 299, L1 (Paper I).

Filippenko, A. (1985). Astron. J. 90, 1172.

Hintzen, P., and Romanishin, W. (1986). Astrophys. J. Lett. 311, L1.

Hu, E., and Cowie, L. (1987). Astrophys. J. Lett. (submitted).

Hutchings, J. (1983). Publ. Astron. Soc. Pac. 95, 799.

Hutchings, J., Hickson, P., and De Robertis, M. (1986). Astron. J. 92, 279.

McCarthy, P., Spinrad, H., Djorgovski, S., Strauss, M., van Breugel, W., and Liebert, J. (1986). Bull. Am. Astron. Soc. 18, 903.

Perley, R. A. (1982a). In Extragalactic Radio Sources, IAU Symposium
No. 97, edited by D. Heeschen and C. Wade (Reidel, Dordrecht), p. 175. Perley, R. A. (1982b). Astron. J. 87, 859.

Perley, R. A. (1986). In Synthesis Imaging, NRAO Workshop No. 13, edited by R. Perley, F. Schwab, and A. Bridle (NRAO, Green Bank, WV).

Schneider, D., Gunn, J., Turner, E., Lawrence, C., Hewitt, J., Schmidt, M., and Burke, B. (1986). Astron. J. 91, 991.

Shara, M., Moffat, A., and Albrecht, R. (1985). Astrophys. J. 296, 399.

Spinrad, H., and Djorgovski, S. (1984). Astrophys. J. Lett. 285, L49.

Spinrad, H., Filippenko, A., Wyckoff, S., Stocke, J., Wagner, M., and Lawrie, D. (1985). Astrophys. J. Lett. 299, L6.

Stockton, A. (1982). Astrophys. J. 257, 33.

Stockton, A., and MacKenty, J. (1983). Nature 305, 678.

Stone, R. (1977). Astrophys. J. 218, 767.

Tyson, J. A. (1986). Astron. J. 92, 691.

Ulvestad, J., and Wilson, A. (1984). Astrophys. J. 285, 439

van Breugel, W., Filippenko, A., Heckman, T., and Miley, G. (1985). Astrophys. J. 293, 83 . 